\title{
Key Node Selection for Containing Infectious Disease Spread Using Particle Swarm Optimization
}

\author{
Xiuju Fu*1, Sonja Lim* ${ }^{*}$ Lipo Wang ${ }^{\dagger}$ \\ Gary Lee*, Stefan $\mathrm{Ma}^{\nabla}$, Limsoon Wong ${ }^{\diamond}$, Gaoxi Xiao ${ }^{\dagger}$ \\ *Institute of High Performance Computing \\ ${ }^{*}$ Singapore 138632 \\ $\dagger$ Department of Electrical and Electronic Engineering \\ $\dagger$ Nanyang Technological University \\ $\nabla$ Ministry of Health, Singapore \\ ${ }^{\diamond}$ School of Computing \\ ${ }^{\diamond}$ National University of Singapore \\ ${ }^{* 1}$ Corresponding author \\ Email: fuxj@pmail.ntu.edu.sg
}

\begin{abstract}
In recent years, some emerging and reemerging infectious diseases have grown into global health threats due to high human mobility. It is important to have intervention plans for containing the spread of such infectious diseases. Among various intervention strategies, screening infected people is an efficient way for evaluating the infection scale and controlling the spread of infectious diseases. Considering the cost in manpower and limited screening machines available, we face to challenges for selecting the optimal nodes (sites) in order to obtain better screening and control effects. In this paper, particle swarm optimization technique is used to determine key nodes for controlling infectious disease spread, through evaluating the number of people captured at each key node. The research example is shown on evaluating the screening control over train stations in Singapore. The optimization algorithm and control concept can be easily extended to large-scale infectious disease control in other kinds of key nodes and in other geographical regions. The selection for optimal control set of the multi objective optimization problem is done using particle swarm optimization. Numerical simulation shows the effectiveness of the proposed algorithm.
\end{abstract}

\section{INTRODUCTION}

With high mobility of people traveling much faster, further and more frequently than in the past, the potential for emerging infectious diseases to spread rapidly in large scale has become a major concern. To better formulate control strategies of outbreaks of infectious diseases, feasible direct interventions include non-pharmaceutical interventions [6] and health-care interventions. In non-pharmaceutical interventions, people take strategies like school closure, travel restrictions, and contact tracing for control. On the other hand, health-care interventions use pharmaceutical materials to contain the spread of infectious diseases. In health-care interventions, vaccination and antiviral or antibiotic agents are used for spread control.

Interventions aimed at preventing and/or containing infectious disease outbreaks are generally expensive. Therefore, they are expected to obtain effective control outcomes espe- cially when resources are limited and conditions are urgent. School closure and restricting travels are considered as useful ways to prevent large-scale spread of infectious diseases. In March 2008, Hong Kong school officials had made an abrupt decision to close all primary schools for 2 weeks, as an effort to block a rising wave of seasonal influenza. The economic cost is high when considering parental workoff when closing schools, and reduced commercial activities due to restrictive travels. Another direct intervention, tracing contacts of infectives is also expensive [7]. If there are already large community or endemic outbreaks, large-scale contact tracing will be required to identify infectives as soon as possible. The cost of mass contact tracing will be increasing dramatically. And with the shortage of health-care workers for implementing unpredictable large-scale contact tracing, it is not feasible and take much longer time which might miss the correct prevention timing.

During the SARS period, contact tracing was made to detect potential infected persons in Singapore. Those had ever contacted with SARS infectives were forced to follow their Home Quarantine Orders. Schools were closed. Public are alert on the highly contagious disease and make necessary self-protection with masks. The prompt action from Singapore government and health agency leads to elimination of SARS quickly. Although the SARS disaster had passed, it leaves open questions like how to better contain or prevent the spread of similar infectious diseases. From 1918 to 1919, the Spanish influenza pandemic swept the globe, and resulted in the deaths of approximately 40 million people. Remembering the past pandemic influenza and SARS, people concern significantly fast mutated influenza as a potential global health threat. Many research work had been carried out to prevent or plan better containment strategies before the disease turns into large scale spread.

Many mathematical models [8][9] are developed to evalu- 
ate the intervention strategies. In those models, intervention strategies including isolation, household quarantine, school or workplace closure, restricting travel are evaluated. However, only when people are confirmed as infected via clinical test, isolation can be targeted for containment. And quarantine is applicable to targets when targets are traced as contacts of infectives. Applying intensive control strategies like contact tracing to containing a large-scale spread at source is impractical as infection will constantly be reseeded around by traveling persons.

For high contagious diseases like SARS or Avian flu, it is impractical containment strategy to trace every recognized infective only. Mass screening is recommended for this situation. The mass screening for containing highly contagious diseases has been proved as efficient and effective. The wellknown measurement taken during SARS period in 2003 is the application of Infrared Fever Sensing System camera set up to help screen passengers with fevers especially arriving from SARS-hit countries at Changi International Airport in Singapore.

Thus, when confronted with large-scale spread of infectious diseases like SARS or new influenza, mass screening at key nodes is a practical containment strategy. Looking worldwide, ports or airports [1][2][3][4] are traditionally candidate key control nodes or sites to screen individuals for safety check and health status in terms of infectious diseases. According to the report [5], the main benefits of screening infection diseases at key points of concentration of moving humans include 1) excluding diseases if the screening systems are sufficiently sensitive and comprehensive 2) detecting disease spread patterns through key nodes in order to give early warnings on the source and help plan post-entry procedures more efficiently 3 ) providing screening data on the national or international movement of diseases to support preparedness and prediction.

In addition, when measuring machines or manpower are limited for screening, it would be more effective if an optimal location subset is select to capture a large percentage of the population and also cover representative sub-population of different regions with low bias. Although mass screening is a practical containment strategy, it may be impractical to screen people door-to-door or even at every node. The question asked is: "what is the best strategy to conduct screening when manpower and screening machines are limited?". It then turns to be an optimization problem. It would be very useful to analyze the optimal key nodes and therefore provide references for health agencies before the outbreak of any infectious disease requiring mass screening. In this paper, we use particle swarm optimization technique to solve the optimization problem.

As a member of swarm intelligence family, particle swarm optimization (PSO) was initially developed by Kennedy and Eberhart [10] in 1995. It is a computationally intelligent optimization method inspired by the social behavior of bird flocking or fish schooling. With roots in artificial life and social psychology as well as in engineering and computer science,
PSO had been applied in various areas [11][12][13]. PSO has many similar characteristics with evolutionary computation techniques. Compared with genetic algorithm (GA), PSO has no operators such as crossover and mutation. In PSO, the potential solutions, called particles, walk through the problem space by following direction of the current optimum solutions.

This paper is organized as follows. Section I introduces the background of key node control optimization. In Section II, we present the detailed optimization model based on particle swarm optimization. In Section III, the experimental results are shown. Finally, in Section IV, we conclude the paper and discuss the analysis results.

\section{Key Node Control Problem Formulation Based ON PARTICLE SWARM OPTIMIZATION}

\section{A. Problem Formulation}

Key node control is a prevention strategy for controlling infectious disease spread in endemic or pandemic scales. Key node control becomes an optimization problem when policy makers want to measure the infection scale and control infectious disease spread with limited manpower and resources (screening equipments, monitoring equipments) in advance. In order to better explain the problem, we focus on key node selection problem for controlling endemic infectious disease spread. In this paper, we look at the key node control in Singapore with Mass Rapid Transit (MRT) train stations as key nodes. If looking at pandemic infectious disease spread world wide, international airports can be considered as key nodes.

Singapore is an island city-state, whose population is around 4.5 million. As an highly urbanized city, suitable locations for screening would be public places such as shopping malls, bus interchanges and Mass Rapid Transit(MRT) train stations, as these are places that a substantial percentage of Singaporean frequently visit. In this work, the focus is on MRT train stations, but the particle swarm algorithm can be applied to any other kinds of nodes. There are more than 1.4 million train rides each day currently. There are totally 64 train stations cross the city. If considering people with double trips usually, there are around 700,000 train passengers daily. The total commuters covered by MRT stations is above $15 \%$ of the total population. Compared with other public locations like bus interchanges or bus stops, the commuter capacity of MRT stations is more concentrated and larger. In addition, the MRT lines across the whole city including North-South, NorthEast and East-West lines now. There are a circle line under developing to link more development guide plan (DGP) zones together. Out of 55 DGP zones in Singapore, only 32 are resident areas. If there are enough manpower and screening machines for mass screening over all MRT stations, as high as $15 \%$ of the total population can be inspected. It is much more efficient than doing door-to-door check or inspect other key concentrated public moving nodes.

However, one concern about high contagious infectious diseases like SARS is that they usually are unpredictable in advance. No one knows when this kind of infectious disease 
spread is going to happen, what is their spread scale, and what is the symptoms, etc.. The screening machines and screening manpower might not be available with enough supply. This calls for research on key node selection optimization. When carrying out screening on infectious diseases with limited resources, we expect to evaluate the spread condition by inspect the representative populations of each DGP zones if considering Singapore scenario. Thus, the constraints have to be taken into consideration to obtain an optimal, practical and feasible solution on key node control include:

- maximizing the number of people screened through limited key node control in order to screen as many as population based on resources available

- balancing the number of people screened among each DGP zones in order to ensure that a representative sample is collected

It is important to get a representative sample of the different residential regions. If an individual is infected, the people living in the same area are a higher chance to be infected. A more representative sample would be helpful in identifying hot spots, and therefore, corresponding containment strategy could be made accordingly.

\section{B. Discrete Particle Swarm Optimization on the Key Node Control Problem}

Optimization problems are set in a space featuring continuous, discrete, or hybrid qualitative distinctions between variables. Among various problems, those scheduling or arranging of discrete elements can be categorized as optimization with discrete variables in the problem space. Besides these combinatorial problems, floating point problems can also be transformed into binary terms, and solve them in a discrete number space.

In a conventional PSO algorithm, the parameters of an optimization problem can be conceptualized as the location of a particle in the problem space. The location is symbolized as $R_{n}$, i.e., the problem space is an $n$-dimensional space of real numbers. The position of a particle $i$ is assigned the algebraic vector symbol $X_{i}$. The particle dynamics is leaded by velocity, which is symbolized by symbol $V_{i}$. Velocity is a vector of numbers that are added to the position coordinates in order to move the particle to the next stage.

$$
X_{i}(t)=X_{i}(t-1)+V_{i}(t)
$$

where $X_{i}$ is the current position of particle $i . V_{i}$ is the velocity of particle $i$ :

$$
V_{i}(t)=V_{i}(t-1)+\varphi_{1}\left(P_{i}+X_{i}(t-1)\right)+\varphi_{2}\left(P_{g}-X_{i}(t-1)\right)
$$

here $P_{i}$ is the particle's own best solution. $P_{g}$ is the neighborhood best. $\varphi_{1}$ and $\varphi_{2}$ are random numbers with a predefined upper limit, with $\varphi_{1}+\varphi_{2}=$ upper limit.

Thus, the particle $i$ moves unevenly around a point defined as a weighted average of the two bests:

$$
\frac{\varphi_{1} P_{i}+\varphi_{2} P_{g}}{\varphi_{1}+\varphi_{2}}
$$

Because of the random numbers, the exact location of this point changes for every iteration [14].

In 1997, Dr James Kennedy and Dr Russell C. Eberhart published a paper detailing a discrete binary version of the particle swarm algorithm [15]. In the space with binary parameters, the particles move in the hypercube by flipping various numbers of bits. The velocity of the particle is described by the number of bits changed per iteration, or the Hamming distance. Therefore, velocity is defined in terms of probability that the bit will flip. The particle moves in a space that is restricted to either one or zero along each dimension. In this work, optimal key nodes are to be selected with a predefined number $M$ of locations which can be controlled based on the capacity of resources.

We have $d$ referring to the index of MRT stations in the discrete PSO equations as follows. $d=0,1,2,3, \cdots, 63$.

$$
\begin{aligned}
V_{i d}(t)= & w\left(V_{i d}(t-1)\right)+\varphi_{1}\left(P_{i d}+X_{i d}(t-1)\right)+ \\
& \varphi_{2}\left(P_{g d}-X_{i d}(t-1)\right)
\end{aligned}
$$

The discrete PSO is similar with continuous PSO. However, $P_{g d}$ and $X_{i d}$ are integers and $V_{i d}$ is constrained to interval $[0.0,1.0]$. According to [16], $w$ is added into PSO equations, which is applicable to both continuous and discrete PSO. In continuous particle swarm optimization, a large $w$ encourages global search while a small $w$ facilitates a more global search. In discrete particle swarm algorithm, the reverse is true.

To constrain the velocity to this interval, the sigmoid function can be used:

$$
S\left(V_{i d}\right)=\left(1+\exp \left(-V_{i d}\right)\right)^{-1}
$$

\section{Fitness Evaluation on Key Node Selection for Infectious} Disease Screening in Singapore

The optimization algorithm should select the screening sites that best fit the constraints. Two criteria were identified as stated in Section II-A including maximizing coverage of people and getting representative samples of DGP resident zones.

The total number of people screened from the selected MRT stations is $T_{D G P}$ :

$$
\begin{aligned}
T_{D G P}(X)= & \sum_{k=1}^{Z} \sum_{d=0}^{N} T_{k d}^{b} X_{d} \\
& +\sum_{k=1}^{Z} \sum_{j=0}^{N} T_{k j}^{a} X_{j} \\
& -\sum_{k=1}^{N} \sum_{d=0}^{N} \sum_{j=0}^{N} T_{k d j}^{a} X_{d} X_{j}
\end{aligned}
$$

here $Z$ is the number of urban planning areas, $Z=32 . N$ refers to the ID of MRT stations. The value of $X_{d}$ corresponds to the status of station $d$ :

- $X_{d}=1$, if station $d$ is selected for screening

- $X_{d}=0$, if station $d$ is not selected

$T_{k d}^{b}$ represents the population boarding at station $d$ from DGP area $k . T_{k j}^{a}$ represents the population coming from DGP area $k$, boarding at some station and alighting at station $j$. In the two items, there are duplicate screenings. For example, 
when both stations $i$ and $j$ are selected for screening ( $X_{i}$ and $X_{j}$ equal to 1 ), the passengers board at station $i$ but alight at station $j$ are counted twice. To removing duplicate counting, the third item $T_{k d j}^{a}$ in the equation is used to remove duplicated counting. $T_{k d j}^{a}$ represents the population coming from area $k$, boarding at station $d$ and alighting at station $j$.

When maximizing $T_{D G P}$, we are going to get maximum coverage of people. However, we also need to ensure that a representative sample is to be screened by reducing the variance in the number of people screened from different regions of Singapore. Hence we have the variance of population screened coming from different residential areas:

$$
\operatorname{Var}(X)=\sum_{i=1}^{Z}\left(P_{i}-P_{\text {ave }}\right)^{2} / 32
$$

here $X=X_{1}, X_{2}, \ldots, X_{N}$ is $P_{i}=\sum_{d=0}^{N} T_{i d}^{b} X_{d}+$ $\sum_{d=0}^{N} T_{i d}^{a} X_{d}$. Similar with Eq. 6, $T_{i d}^{b}$ represents the population coming from from DGP area $i$ and boarding at station $d . T_{i d}^{a}$ represents the population coming from from DGP area $i$ and alighting at station $d$.

Hence, the fitness function is:

$$
F(X)=T_{D G P}(X) / L-\operatorname{Var}(X)
$$

$L$ is the normalization parameter to make the two items in the fitness function with similar scales. $L$ is determined empirically.

\section{Transportation Data}

The data of MRT transportation are simulated due to confidentiality and the lack of practical data. The data simulation is based on the census 2000 data of Singapore,(http://www.singstat.gov.sg/pubn/popn/c2000sr4/

cop2000sr4.pdf) in which the number of people taking MRT transportation mode from each DGP zone is given.

- It was assumed that people taking the MRT to work and school would take the train from MRT stations within their DGP or at the border between two DGPs

- Certain DGPs such as Orchard and Singapore River have few of people who commute to work or school, due to low population density as they are shopping, entertainment and business areas. Therefore, it is assumed that no residential people in the areas.

We could take the screening from afternoon to the evening since residents are coming back from work places and schools and visiting people are leaving to return their homes via train stations. At the screening point, both people boarding and alighting are screened.

When implementing screening, we also need to consider difference of people's moving patterns in weekday and weekends. In an urban city, some regions are mainly used as business purposes, some are mainly used for industrial areas with factories, some are developed as residential areas etc.. There may be remarkably different in weekday and weekend traveling patterns in well-developed urban cities. In this work, we will also check whether screening should be targeted as different site sets for weekday and weekends. Two different traveling data are generated according to main functions of Singapore urban planning areas for weekday and weekend respectively.

\section{EXPERIMENTAL RESULTS}

In Eq. $4, w=0.7 . \varphi_{1}$ and $\varphi_{2}$ and are randomly generated, with the constraint $\varphi_{1}+\varphi_{2}=4$. The sigmoid function keeps the velocity within the range $0<V_{i d}(t)<1$. The particles are initialized by picking $M$ points randomly out of $N=64$ and setting the values to 1 . The velocity is initialized as 0 in all dimensions. In this work, $M$ is set as 10 . That is, assume there are 10 screening equipments available before conducting the screening operation. In Eq. 8, $L=1000.30$ particles are used in this optimization process.

The individual particle in a particle swarm algorithm is influenced by its personal best and the best result found in its neighborhood. The neighborhood can be defined as a subset of the population, in which case it is referred to as 'lbest' or local best, or the entire population itself, 'gbest' or global best. We compare the global best and local best methods. For local best, the neighborhood size was defined as 3 , therefore for particle $\mathrm{i}$ its neighborhood consisted of particle $i-1$, itself and $i+1$. The program was run 100 times each for the weekday data set and weekend data set. Table I shows the results: The two methods

TABLE I

COMPARISONS ON LOCAL BEST AND GLOBAL BEST STRATEGIES IN PSO

\begin{tabular}{|l|c|c|c|c|}
\hline \multirow{2}{*}{ Comparisons } & \multicolumn{3}{|c|}{ Localbest } & \multicolumn{2}{c|}{ Globalbest } \\
\cline { 2 - 5 } & weekday & weekend & weekday & weekend \\
\hline average fitness & 184.5 & 175.2 & 186.05 & 175.2 \\
\hline $\begin{array}{l}\text { average number } \\
\text { of iterations }\end{array}$ & 1883 & 1907 & 1833 & 1788 \\
\hline best fitness found & 194.37 & 183.67 & 194.38 & 185.32 \\
\hline worst fitness found & 179.43 & 167.6 & 179.43 & 170.08 \\
\hline $\begin{array}{l}\text { standard deviation in } \\
\text { fitness for 100 runs }\end{array}$ & 3.506 & 3.167 & 3.757 & 3.094 \\
\hline $\begin{array}{l}\text { average computational } \\
\text { time per run(secs) }\end{array}$ & 7.985 & 5.125 & \\
\hline
\end{tabular}

are comparable. The global best method shows slightly better results in the weekday average fitness and faster computational time, as globalbest causes the particles to have greater velocity and make greater leaps across the problem space. Figure 1 shows the convergence of the BPSO algorithm.

Since people are usually alight via different MRT stations at weekday and weekend for different purposes, we simulate the traveling patterns according to the function (working areas or business areas) of linking areas of MRT stations. Based on experimental results, the number of people screened during weekend will be less than the number screened at weekday if monitoring the same 10 MRT sites selected for weekday. Hence, it is suggested to carry out at different site sets in order to get better screening effect for weekday and weekend based on traveling patterns. 


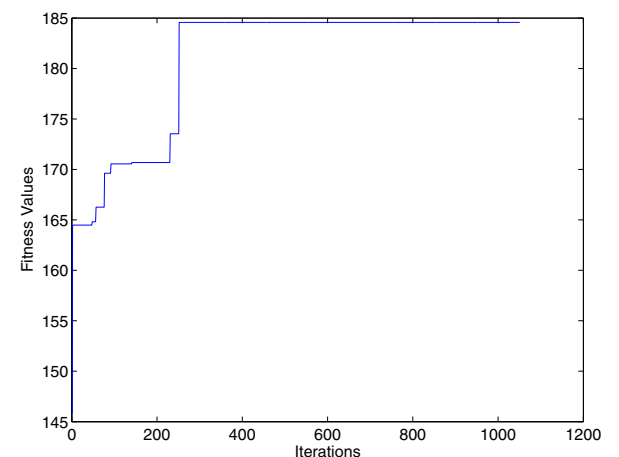

Fig. 1. Convergence of BPSO algorithm

\section{CONCLUSIONS}

PSO is shown useful for determining the key node control sites for mass screening based on the traffic data of MRT data in this paper. In an early stage of the spread of an infectious disease, the mass screening could help to sift mass population efficiently and alert the people at key nodes who are also those with high degree in the whole contact network. At later stage, mass screening could help to evaluate the overall infectious situation, and check the infectious situation in individual residential areas, which facilitate and direct health agency to target at hot spots.

Although the simulated data may vary from practical transportation data, the controlling concept and the optimization algorithm can be applied directly to deliver the more practical screening sites when practical data are input in the optimization model. The selected sites may vary when actual traffic flow data are used. This research work not only targets at determining optimal locations for infectious disease spread, but also emphasize the role of optimization concept on containing infectious disease spread worldwide if considering the key nodes or locations as international airports or other key nodes. It would be useful to explore the problem formalization and the optimization algorithm in case there is any kind of practical challenges beforehand.

\section{REFERENCES}

[1] Colizza V., Barrat A., Barthlemy M., Vespignani A., "The role of the airline transportation network in the prediction and predictability of global epidemics"', Proc Natl Acad Sci U S A. 2006 Feb 14;103(7):2015-20.

[2] Colizza V., Barrat A., Barthelemy M, Valleron AJ, Vespignani A, "Modeling the Worldwide Spread of Pandemic Influenza: Baseline Case and Containment Interventions", PLoS Medicine Vol. 4, No. 1, e13 doi:10.1371/journal.pmed.0040013

[3] Guimera R., Mossa S., Turtschi A., Amaral LA. "The worldwide air transportation network: anomalous centrality, community structure, and cities global roles". Proc Natl Acad Sci U S A. 102: 77949

[4] Hollingsworth T.D., Ferguson N.M., Anderson R.M., "Frequent travelers and rate of spread of epidemics". EMERG INFECT DIS. 13:1288-1294, 2007

[5] http://www.foresight.gov.uk/previous_projects/detection_and_ identification_of_infectious_diseases/Reports_and_Publications /Final_Reports/D/d2_4.pdf

[6] Mossong J., Hens N., Jit M., Beutels P., Auranen K., et al., "Social Contacts and Mixing Patterns Relevant to the Spread of Infectious Diseases", PLoS Medicine Vol. 5, No. 3, e74 doi:10.1371/journal.pmed.0050074, 2008
[7] Gustavo H. Dayan, Ismael R. Ortega-Snchez, Charles W. LeBaron, M. Patricia Quinlisk and the Iowa Measles Response Team, "The Cost of Containing One Case of Measles: The Economic Impact on the Public Health Infrastructure - owa 2004", Pediatrics, Jul 2005; 116: e1 - e4.

[8] G. Chowell, et al., Model parameters and outbreak control for SARS, Emerging Infectious Diseases 10 (7) (2004) 12581263.

[9] Neil M. Ferguson, Derek A. T. Cummings, Christophe Fraser, James C. Cajka, Philip C. Cooley and Donald S. Burke, "Strategies for mitigating an influenza pandemic", Nature 442, 448-452 (27 July 2006)

[10] J. Kennedy and R. C. Eberhart, Particle swarm optimization,Proc. IEEE Int. Conf. Neural Netw. Perth, Australia, vol. IV, 1995, pp. 1942-1948.

[11] Fu-Qing Zhao; Qiu-Yu Zhang; Ya-Hong Yang; "A Hybrid Self-Adaptive Pso Algorithm and its Applications for Partner Selection in Holonic Manufacturing System (HMS)", 2006 International Conference on Machine Learning and Cybernetics, Aug. 2006 Page(s):2536 - 2541

[12] Cui, S.; Weile, D.S.; "Application of a parallel particle swarm optimization scheme to the design of electromagnetic absorbers", IEEE Transactions on Antennas and Propagation, Vol. 53, No. 11, Nov. 2005 Page(s):3616 - 3624

[13] Venayagamoorthy, G.K.; Doctor, S.; "Navigation of mobile sensors using PSO and embedded PSO in a fuzzy logic controller", Industry Applications Conference, Vol. 2, Page(s):1200 - 1206, 3-7 Oct. 2004

[14] Kennedy J. and Eberhart R. C. with Y. Shi, Swarm Intelligence, Morgan Kaufmann Publisher, 2001

[15] Eberhart, R C and Kennedy, J, 1997, 'A Discrete Binary Version of the Particle Swarm Algorithm', Systems, Man and Cybernetics, 1997. IEEE International Conference on 'Computational Cybernetics and Simulation'., Volume 5, pp 4104-4108

[16] Eberhart, R C and Shi Y, 1999, 'Empirical Study of Particle Swarm Optimization', Proceedings of the 1999 Congress on Evolutionary Computation, CEC 99, Volume 3, pp 1950 